\title{
Análise das principais manifestações oculares de pacientes hansenianos nas regiões Norte e Sudeste do Brasil
}

\author{
Analysis of the main ocular presentations in leprosy \\ patients in Northern and Southeastern Brazil
}

Rodrigo Angelucci', Paulo Sampaio², Rodrigo Proto ${ }^{3}$, Lúcia Sato ${ }^{4}$, José Ricardo Rehder

\begin{tabular}{|c|}
\hline Resumo \\
\hline $\begin{array}{l}\text { Objetivo: Analisar as principais manifestações oculares encontradas em pacientes di- } \\
\text { agnosticados com Hanseníase nas regiões Norte e Sudeste do Brasil. Métodos: Foram } \\
\text { examinados } 28 \text { pacientes com diagnóstico de hanseníase, sendo } 12 \text { pacientes - Grupo I, } \\
\text { da região Sudeste (ABC) e } 16 \text { pacientes - Grupo II, da região Norte (Amazônica) do } \\
\text { país. O Grupo I foi observado no período de agosto de } 2003 \text { a junho de } 2004 \text { no Ambu- } \\
\text { latório de Oftalmologia da FMABC-SP e o segundo grupo na } 10^{a} \text { missão do Projeto } \\
\text { Amazônia Visão } 2000 \text { realizada no mês de julho de } 2004 \text {. Todos os } 28 \text { pacientes foram } \\
\text { submetidos a exame oftalmológico completo. Resultados: Em relação ao sexo notou-se } \\
\text { em ambos os grupos predomínio do sexo masculino. Observou-se um predomínio de } \\
\text { indivíduos de } 50 \text { a } 60 \text { anos na região amazônica e de } 40 \text { a } 50 \text { anos na região do ABC. } \\
\text { Quanto à forma clínica da doença observaram-se na região amazônica } 13 \text { casos ( } 81 \% \text { ) } \\
\text { de hanseníase virchowiana (MHV) e } 3 \text { casos (19\%) de hanseníase tuberculóide (MHT). } \\
\text { Na região do ABC } 6 \text { pacientes (50\%) foram diagnosticados com hanseníase dimorfa } \\
\text { (MHD), } 5 \text { ( } 41 \% \text { ) hanseníase virchowiana e apenas } 1 \text { ( } 9 \% \text { ) hanseníase tuberculóide. } \\
\text { Não foram encontradas formas de hanseníase indeterminada (MHI) nas } 2 \text { regiões } \\
\text { investigadas, sugerindo que em ambas, o diagnóstico foi tardio. Conclusões: Neste estu- } \\
\text { do verificou-se um predomínio das alterações oculares com maior gravidade nos paci- } \\
\text { entes examinados na região amazônica. Encontraram-se alterações oculares principal- } \\
\text { mente nas formas clínicas mais graves da doença. }\end{array}$ \\
\hline
\end{tabular}

Descritores: Hanseníase; Baixa visão; Cegueira/etiologia; Amazônia

\footnotetext{
Colaborador do Setor de Retina e Vítreo da Faculdade de Medicina do ABC - FMABC - Santo André (SP), Brasil. ${ }^{2}$ Chefe do Setor de Visão Subnormal da Oftalmologia da Faculdade de Medicina do ABC - FMABC - Santo André (SP), Brasil. ${ }^{3}$ Auxiliar de Ensino da Disciplina de Dermatologia da Faculdade de Medicina do ABC - FMABC - Santo André (SP), Brasil. ${ }^{4}$ Professora Auxiliar de Ensino da Disciplina de Dermatologia da Faculdade de Medicina do ABC - FMABC - Santo André (SP), Brasil. ${ }^{5}$ Professor Titular da Disciplina de Oftalmologia da Faculdade de Medicina do ABC - FMABC - Santo André (SP), Brasil ; Professor Adjunto da Universidade Federal de São Paulo - UNIFESP - São Paulo (SP), Brasil.

Trabalho realizado no Departamento de Oftalmologia, Departamento de Dermatologia da Faculdade de Medicina do ABC - FMABC - Santo André (SP), Brasil e durante a 10ª missão do Projeto Amazônia Visão 2000.

Recebido para publicação em: 01/03/07 - Aceito para publicação em 13/08/07
} 


\section{INTRODUÇão}

A Hanseníase é uma das doenças mais antigas da humanidade, sendo evidenciada em descrições de povos antepassados como Egípcios e Romanos. Disseminou-se pela Europa durante as cruzadas na Idade Média e desde àquela época, até os dias atuais, os indivíduos acometidos pela enfermidade são freqüentemente isolados, excluídos e abandonados por suas famílias e pela sociedade ${ }^{(1-3)}$

Atualmente é considerada como um problema de saúde pública apresentando-se de forma endêmica nas áreas tropicais e subtropicais do planeta, estando o Brasil incluído nesta realidade ${ }^{(3.5)}$.

A doença atingiu a Amazônia brasileira pelo estado do Pará, no início do século XIX, e o restante do país com a intensa imigração para os diversos estados, incluindo as regiões Sul e Sudeste ${ }^{(6-7)}$. Nos dias atuais o Estado do Amazonas, com uma prevalência de 33,29/10.000 habitantes, é o segundo Estado mais acometido pela doença no país sendo considerado como zona hiper-endêmica ${ }^{(8)}$.

Esta doença caracteriza-se por uma infecção granulomatosa crônica causada pelo Mycobacterium leprae que afeta, principalmente, os tecidos ou órgãos com temperaturas mais baixas, tais como, nervos superficiais, extremidades da pele (nariz, falanges, orelhas) e globo ocular podendo resultar em quadros mutilantes com seqüelas irreversíveis.

Em 1873, Bull e Hansen já descreveram as complicações oculares dos pacientes com hanseníase ${ }^{(2)}$.

As lesões oculares nos pacientes com Hanseníase podem ser classificadas em 4 categorias ${ }^{(9)}$ :

1. Lesões por invasão direta do M. leprae;

2. Reações inflamatórias como conseqüência de formação de imunocomplexos;

3. Lesões secundárias ao comprometimento dos nervos cranianos (principalmente V e VII pares);

4. Lesões secundárias à reação granulomatosa dos tecidos perioculares.

Baixa de visão e cegueira são algumas das alterações oculares relatadas da doença.A frequiência de acometimento dessas alterações pode variar de 20 a $80 \%$ de acordo com o tempo decorrido até o diagnóstico, formas clínicas, inabilidade político-social e pelo ostracismo ao hanseniano limitando o acesso de tratamento adequado ${ }^{(10)}$.

Estima-se que existam, aproximadamente, 250 mil pacientes em todo o mundo com déficit visual grave por lesões oculares secundárias à hanseníase. Pelo menos $1 / 3$ dos pacientes irão desenvolver algum tipo de comprometimento ocular durante a evolução da doença ${ }^{(11)}$.

As seqüelas desse mal ("Mal de Hansen") são pas- síveis de medidas preventivas, que beneficiariam imensamente os portadores diretos e indiretos desta terrível doença e com certeza, o custo destes programas seria muito menor para a comunidade do que o custo representado por indivíduos portadores de cegueira evitável ${ }^{(12)}$.

Desde 1997 o Projeto Amazônia Visão 2000, coordenado pela Disciplina de Oftalmologia da Faculdade de Medicina do ABC - SP em parceria com o Governo e iniciativa privada tem levado à população ribeirinha do Rio Amazonas e aos grupos de aldeamento Indígena da região um programa de Saúde Ocular e Prevenção da Cegueira.

O objetivo deste estudo é analisar as principais manifestações oculares encontradas em pacientes diagnosticados com Hanseníase nas regiões Norte e Sudeste do Brasil.

\section{Métodos}

Foram examinados 28 pacientes com diagnóstico de hanseníase confirmado por médicos dermatologistas da Disciplina de Dermatologia da FMABC, sendo 12 pacientes - Grupo I, da região Sudeste $(\mathrm{ABC})$ e 16 pacientes - Grupo II, da região Norte (Amazônica) do país.

$\mathrm{O}$ primeiro grupo de pacientes (Grupo I) faz acompanhamento e tratamento regular da doença no Ambulatório de Hanseníase da Disciplina de Dermatologia da FMABC - SP. O segundo grupo (Grupo II) compõe-se de pacientes ribeirinhos da região do Rio Purús (cidades de Lábria, Tapauá, Canutama e Belo Monte) - AM que foram examinados por um médico dermatologista da FMABC - SP integrante de uma das missões do Projeto Amazônia Visão 2000. Os pacientes do grupo II encontram-se em acompanhamento pós-alta nas respectivas localidades e a coleta dos dados foi orientada pelos agentes de saúde locais.

O Grupo I foi observado no período de agosto de 2003 a junho de 2004 no Ambulatório de Oftalmologia da FMABC-SP e o segundo grupo na $10^{\mathrm{a}}$ missão do Projeto realizada no mês de julho de 2004.

Este projeto, instituído em 1997, utiliza 3 navios de assistência hospitalar (NASH) do Ministério da Saúde, NASH Carlos Chagas, NASH Oswaldo Cruz e CCO Montenegro que são coordenados pelo $4^{\circ} \mathrm{Comando} \mathrm{Na}-$ val da Marinha Brasileira e que desde 1984 desenvolvem atividades assistenciais em Medicina, Odontologia e ações preventivas na região.

A parceria facilita o trabalho da equipe do projeto, que tem condições de chegar com maior facilidade, com o uso de lanchas e helicóptero, às localidades mais distantes das margens do rio.

Os navios são transformados em Hospital 
oftalmológico flutuante com equipamentos de última geração na área da Oftalmologia com o intuito de executar desde consultas e orientações preventivas até as mais complexas cirurgias e tratamentos oftalmológicos.

Todos os 28 pacientes foram submetidos a exame oftalmológico completo, sendo no Grupo I realizado no Ambulatório do Instituto de Olhos da Disciplina de Oftalmologia da Faculdade de Medicina do ABC-SP e no grupo II no NASH Oswaldo Cruz.

O exame consistiu de:

Anamnese, acuidade visual (Snellen), ectoscopia, biomicroscopia, refração, tonometria de aplanação de Goldmann, oftalmoscopia direta e indireta após midríase.

\section{Análise estatística}

Foi feita a análise descritiva de todas as variáveis do estudo. As variáveis foram apresentadas em termos de seus valores absolutos e relativos.

Para a verificação da associação entre grupos, sexo e formas clínicas utilizou-se o teste exato de Fisher.

Para avaliar as diferenças entre as proporções de doenças oculares com as formas clínicas de hanseníase entre as duas regiões foi aplicado o teste de diferença de proporção. O nível de significância foi de $10 \%$.

\section{Resultados}

Em relação ao gênero notou-se no grupo I um predomínio de 7 homens e 5 mulheres e no grupo II 10 homens e 6 mulheres (Tabela 1).

Quando avaliada a faixa etária dos pacientes observamos um predomínio de indivíduos de 50 a 60 anos na região amazônica e de 40 a 50 anos na região do ABC (Gráfico 1).

Quanto à forma clínica da doença observaram-se na região amazônica 13 casos $(81 \%)$ de Hanseníase virchowiana (MHV) e 3 casos (19\%) de Hanseníase tuberculóide (MHT). Na região do ABC 6 pacientes (50\%) foram diagnosticados com Hanseníase dimorfa (MHD), 5 (41\%) Hanseníase virchowiana e apenas 1 (9\%) Hanseníase tuberculóide. Não foram encontradas formas de hanseníase indeterminada (MHI) nas duas regiões investigadas.

Pelo teste de diferença de proporção pode-se observar que na região amazônica há uma proporção maior e estatisticamente significativa de pacientes com Hanseníase virchowiana do que na região do $\mathrm{ABC},(\mathrm{p}<$ 0,05), como demonstrado no Gráfico 2:

Pelo teste de Fisher observou-se que na região amazônica há uma incidência maior e estatisticamente significativa de pacientes com alterações
Tabela 1

Distribuição numérica dos 2 grupos de pacientes por gênero

\begin{tabular}{l|cccc}
\hline \multirow{2}{*}{ Sexo } & \multicolumn{2}{c}{ ABC } & \multicolumn{2}{c}{ Amazônia } \\
\hline Masculino & $N^{\circ}$ & $\%$ & $N^{\circ}$ & $\%$ \\
\cline { 2 - 5 } Feminino & 7 & 65 & 10 & 65 \\
Total & 5 & 35 & 6 & 35 \\
& $\mathbf{1 2}$ & $\mathbf{1 0 0}$ & $\mathbf{1 6}$ & $\mathbf{1 0 0}$ \\
\hline
\end{tabular}

Tabela 2

Incidência das manifestações oculares nos 2 grupos

\begin{tabular}{|c|c|c|c|c|}
\hline Grupo & & & & bnia \\
\hline oculares & $\mathbf{N}^{\mathbf{o}}$ & $\%$ & $\mathbf{N}^{\mathbf{o}}$ & $\%$ \\
\hline Presente & 7 & 65 & 16 & 100 \\
\hline Ausente & 5 & 35 & 0 & 0 \\
\hline Total & 12 & 100 & 16 & 100 \\
\hline
\end{tabular}

Fisher $\mathrm{p}=0,008$

No grupo I, 6 pacientes apresentavam a forma dimorfa, destes 1 apresentava ceratite, 1 leucoma corneano e 4 não tinham alterações; 5 pacientes apresentavam a forma virchowiana, sendo que 2 tinham ceratite, 1 madarosis, 1 trichíase e 1 não tinha alterações; 1 paciente apresentava a forma tuberculóide com madarosis.

No grupo II, 13 pacientes apresentaram-se com a forma clínica virchowiana, sendo que 2 tinham ceratite, 4 lagoftalmo, 3 leucoma corneano, 1 ectrópio, 1 entrópio, 2 úlcera de córnea; 3 pacientes apresentavam a forma clínica tuberculóide sendo 1 com lagoftalmo, 1 com leucoma e 1 com ectrópio. (Gráfico 3)

Dos 28 pacientes avaliados, 23 não apresentaram alterações de segmento posterior à oftalmoscopia indireta. $\mathrm{O}$ exame tornou-se impossível pela opacidade corneana em 5 pacientes.

\section{Discussão}

Nos dois grupos avaliados observou-se maior freqüência de acometimento da doença no sexo masculino, sendo no grupo I a incidência de $58 \%$ e no grupo II $62 \%$ o que corresponde aos resultados de diversos estudos da literatura mundial ${ }^{(1,3,13)}$. Na literatura nacional foi observado uma incidência de $63 \%$ de pacientes do sexo masculino e $27 \%$ do sexo feminino ${ }^{(14)}$. 


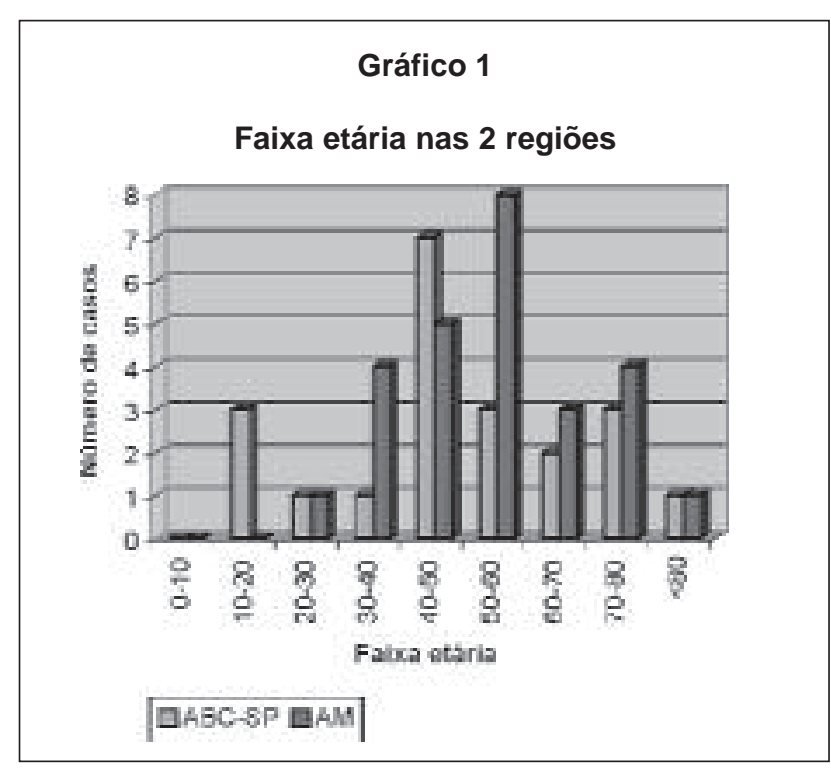

Em relação aos grupos etários da presente amostra a maior incidência de indivíduos acometidos encontrou-se na faixa entre 40-60 anos corroborando os estudos que encontraram uma média de 62,1 anos de idade (1) e outro que observaram uma média de 67,4 anos em 74 portadores da enfermidade ${ }^{(15)}$.

Ocorreu um predomínio de hanseníase da forma virchowiana (81\%) sobre a tuberculóide (19\%) na região Amazônica como descrito por Pinheiro Jr. et al. ${ }^{1}$ que encontraram $84,9 \%$ e $64,7 \%$ desta forma clínica em 2 grupos de pacientes estudados na região de ManausAM. No ABC, as porcentagens de hanseníase tuberculóide, virchowiana e dimorfa foram respectivamente: $9 \%$, $41 \%$ e $50 \%$. Não foram encontradas formas indeterminadas nas duas regiões investigadas.

Uma possível explicação para o maior número de casos virchowianos na Amazônia é o diagnóstico tardio da enfermidade que contribui para o desenvolvimento de quadros seqüelares e incapacitantes. (Figuras 1-3). No mesmo sentido, tal constatação pode explicar a ausência de casos indeterminados tratados ou em tratamento, já que essa forma é considerada como fase inicial da doença.

A região do Grande $\mathrm{ABC}$, por ser um pólo industrial marcado pela alta taxa de migração de indivíduos de outras regiões do país, principalmente do Norte e Nordeste, apresenta a maioria casos de pacientes com hanseníase não diagnosticados no seu local de origem. Por estas razões, quando atendidos no ambulatório de hanseníase da FMABC estes pacientes já se encontram nas fases avançadas da doença muito embora, ao contrário da região Amazônica, nem todos apresentam lesões irreversíveis.

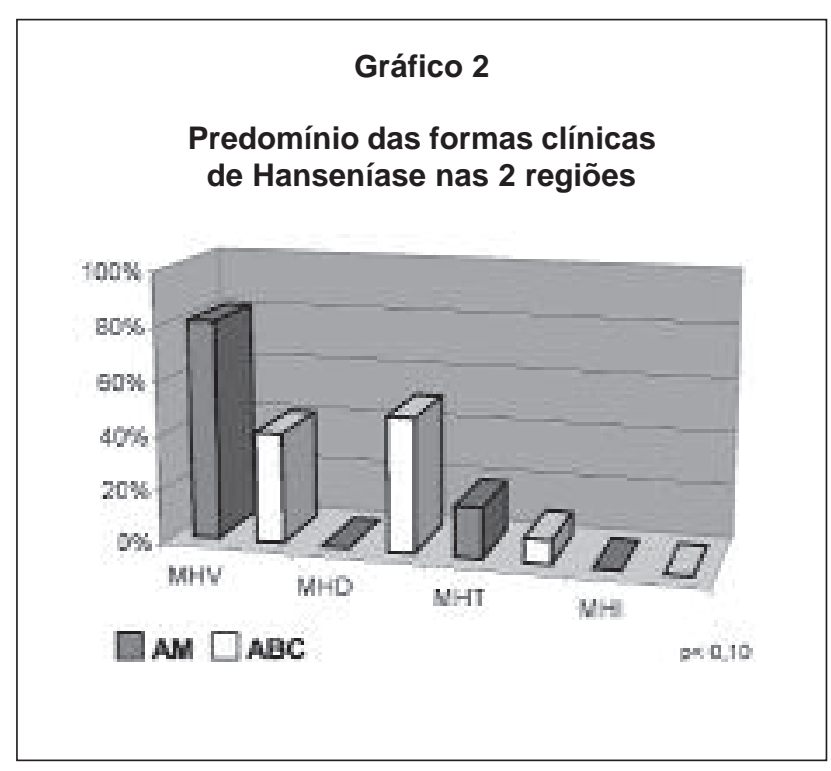

Gráfico 3

Comparação da proporção das manifestações oculares nos 2 grupos

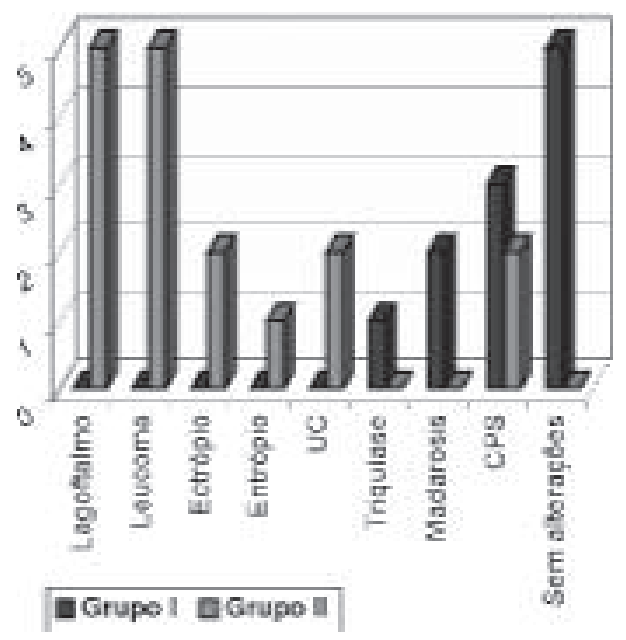

Além disso, a região do Grande ABC é atualmente considerada como zona livre de Hanseníase já que apresenta um coeficiente de prevalência menor que 1/ 10000 habitantes.

As manifestações oculares encontradas nos 28 pacientes estudados coincidem com as descritas como de maior incidência em diferentes estudos ${ }^{\left(\frac{3.12-15)}{4}\right.}$.

Lagoftalmo, úlcera de córnea e leucoma corneano acarretando em baixa de visão permanente foram encontrados com maior freqüência nos casos virchowianos da região amazônica como observado em estudo que 


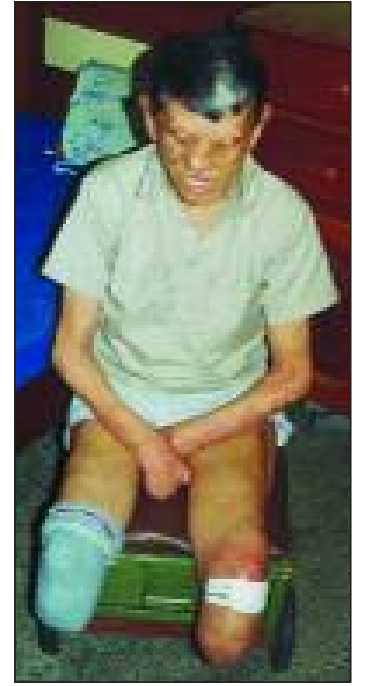

Figura 1: Seqüelas sistêmicas

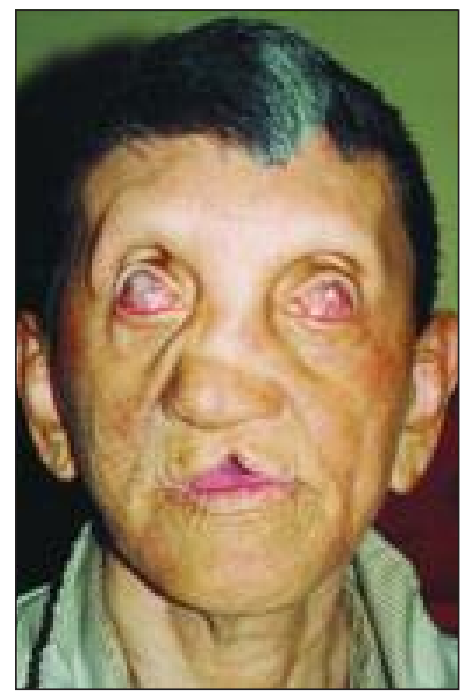

Figura 2: Fácies Leonina com comprometimen to ocular

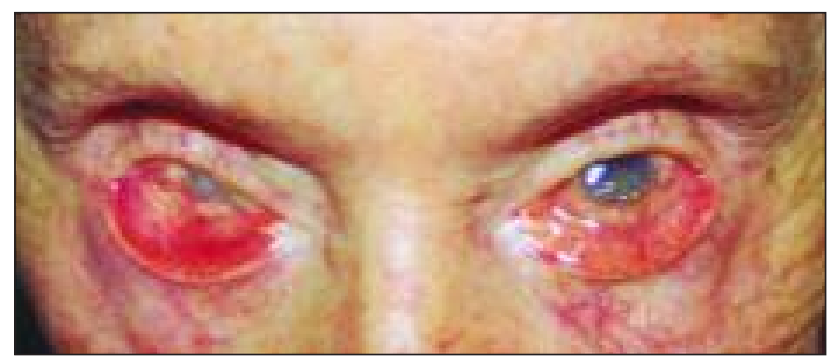

Figura 3: Lagoftalmo com queratinização conjuntival

mostrou resultados de maior gravidade das lesões nas formas clínicas dimorfa e virchowianas reforçando a correlação entre forma clínica e desenvolvimento das seqüelas ${ }^{(15)}$.

Não foram observadas lesões de segmento posterior nos grupos analisados corroborando os achados na literatura compulsada. Isto não significa inexistência de acometimento ocular posterior pela Hanseníase, já que diferentes manifestações clínicas neste segmento do olho já se encontram descritas na literatura mundial ${ }^{(16-20)}$. Tal fenômeno é explicado pela diferença de temperatura entre os segmentos anterior e posterior do globo ocular, já que as estruturas da câmara anterior são cerca de 4 a 6 graus Celsius mais frias que o segmento posterior, $\mathrm{o}$ que propicia melhores condições para a proliferação do bacilo ${ }^{(19)}$.

Deve-se salientar que quando avaliadas nos 2 grupos, as manifestações oculares tenderam a se apresentar com maior gravidade nos pacientes da região amazônica. Por esta razão, se torna plausível a idéia de que associada ao diagnóstico tardio da doença com conseqüente desenvolvimento de formas clínicas mutilantes exista uma mai- or precariedade de recursos sociais na localidade quando comparados à região sudeste do Brasil. Tal fato é reforçado pelos resultados dos 6 anos de atendimento oftalmológico realizados pelo Projeto Amazônia Visão 2000 que demonstram altos índices de alterações oculares como ametropias, catarata, pterígio e glaucoma evidenciando a insalubridade ocular da população amazônica bem como o abandono social, desinformação e inexistência de atendimento oftalmológico adequado na região ${ }^{(20)}$.

Este cenário também é evidenciado em diversos estudos de países subdesenvolvidos da África, Ásia e América Latina que juntos obtém os maiores índices de prevalência de casos registrados de Hanseníase, bem como suas repercussões orgânicas e psicossociais ${ }^{(3,9,14,17,21)}$.

Neste contexto, a necessidade de se implementar programas de prevenção à cegueira, englobando ações de promoção da saúde ocular e prevenção de distúrbios oftalmológicos nesta região do país é evidente e se enquadram perfeitamente na estratégia geral de atenção primária.

Desde sua implantação em 1997, o Projeto Amazônia Visão 2000 mostrou que a parceria entre governo, universidade e empresa privada é um modelo de atendimento eficiente e viável para a prevenção e resolução dos difíceis e complexos problemas da saúde pública ocular brasileira.

\section{Conclusões}

1. Neste estudo verificou-se um predomínio das alterações oculares mais graves nos pacientes examinados na região amazônica.

2. Neste estudo encontraram-se alterações oculares com maior gravidade principalmente nas formas clínicas polarizadas da doença, nas quais o diagnóstico da moléstia foi realizado tardiamente, quando as seqüelas físicas e incapacitantes já estavam presentes.

3. Lagoftalmo, úlcera de córnea e leucoma foram as seqüelas mais observadas nas formas virchowianas da doença.

\section{Abstract}

Objective: To analyse the main ocular presentations observed in leprosy diagnosed patients in Northern and Southeastern in Brasil regions.Methods: Twenty-eight diagnosed leprosy patients were examined divided in two groups. Group I, compound by 12 patients at southeastern $(A B C)$ and group II, compound by 16 patients at northern region (Amazon). Group I was examined from August 2003 to June 2004 at SP, in FMABC ophthalmology 
outpatient service and the second group was examined during the $10^{\text {th }}$ Mission of 2000 Vision Amazon Project that was carried out on July 2004. The twenty-eight patients were submitted to complete ophthalmological trial. Results: It was noted predominance of male in both groups. It was noted the predominance of 50 to 60 years old individuals in Amazonian region and individuals aged 40 to 50 years old in $A B C$ region. Concerning the clinical form of the disease, it was noted that, in Amazon region, there were 13 cases (81\%) of Lepromatous leprosy and 3 cases (19\%) of Tuberculoid leprosy. At ABC region there were 6 patients $(50 \%)$ diagnosed with borderline leprosy, 5 (41\%) lepromatous leprosy and only one (9\%) with tuberculoid leprosy. There weren't forms of indeterminated leprosy in both investigated regions, what suggests a late diagnosis. Conclusion: It was verified a predominance of the most severe ocular changings among the examined patients at Amazonian region. It was observed ocular changings, mainly in most severe clinical forms of the disease. Amazonia

Keywords: Leprosy; Vision,low; Blindness/etiology;

\section{REFERÊNCIAS}

1. Pinheiro Junior MN, Cohen JM. Causas de baixa da acuidade visual em hansenianos de duas instituições de saúde de Manaus. Rev Bras Oftalmol. 2003; 62(2):109-18.

2. Marmor MF. The ophtalmic trials of G.H. A. Hansen. Surv Ophthalmol. 2002; 47(3):275-87.

3. Mvogo CE, Bella-Hiag AL, Ellong A, Achu JH, Nkeng PF. Ocular complications of leprosy in Cameroon. Acta Ophthalmol Scand. 2001; 79(1):31-3.

4. World Health Organization. Leprosy: in point of fact, $n^{\circ} 82$. Genebra; 1994.

5. Leprosy - global situation. Wkly Epidemiol Rec. 2000; 75(28):226-31.

6. Souza Araújo HC. História da lepra no Brasil. Rio de Janeiro: Imprensa Nacional; 1946. v.1.

7. Cohen JM. Estudo epidemiológico das alterações oculares em hansenianos no Amazonas [tese]. Ribeirão Preto: Faculdade de Medicina de Ribeirão Preto da Universidade de São Paulo; 1996.
8. Carvalho RAC, Melo A, Cohen J, Chaves C. Estudo da variação da pressão intra-ocular em hansenianos. Rev Bras Oftalmol. 2003; 62(2):127-31.

9. Job CK, Ebenezer GJ, Thompson K, Daniel E. Pathology of eye in leprosy. Indian J Lepr. 1998; 70(1):79-91.

10. Sundar Rao PS, Daniel E, Kurian N, Gayathri KJ. Epidemiological aspects of ocular morbidity in leprosyaffected persons. Indian J Lepr. 1998; 70(1):115-22.

11. Brasil. Ministério da Saúde. Secretaria de Vigilância em Saúde. Departamento de Vigilância Epidemiológica. Manual de condutas para complicações oculares. Brasília: Ministério da Saúde; 2003. 60p. (Série J. Cadernos de Reabilitação em Hanseníase, 4).

12. Monteiro LG, Campos WR, Orefice F, Grossi MA. Study of ocular changes in leprosy patients. Indian J Lepr. 1998; 70(2):197-202.

13. Oréfice F, Boratto LM. Clinical ocular study in leprosy patients at a sanitary dermatological hospital in Brazil. Indian J Lepr. 1998; 70(2):189-95.

14. Guimarães FC, Cruz AA. Eyelid changes in long-standing leprosy. Ophthal Plast Reconstr Surg. 1998; 14(4):239-43.

15. Courtright P, Kim SH, Lee HS, Lewallen S. Excess mortality associated with blindness in leprosy patients in Korea. Lepr Rev. 1997; 68(4):326-30.

16. Dogra A, Aggarwal A, Bajwa GS, Khurana S. Study of pattern of ocular changes in different types of leprosy patients. Int $\mathrm{J}$ Lepr Other Mycobact Dis. 2002; 70(2):127-9

17. Thompson K, Daniel E. Management of ocular problems in leprosy. Indian J Lepr. 1998; 70(3):295-315.

18. Jiang J, Wei XY. The current situation of lagophthalmos and keratopathy of leprosy in PR China. Indian J Lepr. 1998; 70(1):71-7.

19. Oréfice F, Miranda D, Boratto LM. Encontro de bacilos da doença de Hansen na conjuntiva, corpo vítreo e retina em um olho enucleado de paciente portador da forma Virchowiana. Arq Bras Oftalmol. 1990; 53(1):17-9.

20. Rehder RJ, Sobral Neto H, Carvalho F, Lima VL, Pereira R, Barreiro J, Angelucci RI. Prevalência e causas de cegueira e baixa de acuidade visual entre grupos indígenas da Amazônia legal. Arq Méd ABC. 2001; 25(2):59-62.

21. Lewallen S, Courtright P. Blindness in Africa: present situation and future needs. Br J Ophthalmol. 2001; 85(8):897-903.

END. PARA CORRESPONDÊNCIA:

Rodrigo I. Angelucci

Av. Bosque da Saúde, 546, ap 151, Saúde,

Cep- 04142-081

E-mail: rodrigoangelucci@yahoo.com.br 